\title{
A continuous flaring- to normal-branch transition in Scorpius X-1
}

\author{
P. Casella ${ }^{1,2}$, T. Belloni ${ }^{1}$, and L. Stella ${ }^{2}$ \\ 1 INAF - Osservatorio Astronomico di Brera, via E. Bianchi 46, 23807 Merate (LC), Italy \\ e-mail: casella@merate.mi .astro.it \\ 2 INAF - Osservatorio Astronomico di Roma, via di Frascati 33, 00040 Monte Porzio Catone (Roma), Italy
}

Received 21 February 2005 / Accepted 8 June 2005

\section{ABSTRACT}

We report the first resolved rapid transition from a Flaring Branch Oscillation to a Normal Branch Oscillation in the RXTE data of the Z source Sco X-1. The transition took place on a time scale of $\sim 100 \mathrm{~s}$ and was clearly associated to the Normal Branch-Flaring Branch vertex in the color-color diagram. We discuss the results in the context of possible association of the Normal Branch Oscillation with other oscillations known both in Neutron-Star and Black-Hole systems, concentrating on the similarities with the narrow 4-6 Hz oscillations observed at high flux in Black-Hole Candidates.

Key words. accretion, accretion disks - X-rays: binaries - stars: individual: Sco X-1

\section{Introduction}

Neutron-star Low Mass X-ray Binaries are usually classified in two classes, the $\mathrm{Z}$ and the atoll sources; these names are based on the shape that individual sources describe in the color-color diagrams as a result of changes taking place in the sources (CDs, Hasinger \& van der Klis 1989; van der Klis 2005). The atoll sources trace a pattern consisting of a single curved branch (banana branch), together with a smaller "island", while the pattern traced by $\mathrm{Z}$ sources is characterized by three branches called horizontal branch, normal branch, and flaring branch, respectively. Three types of Low-frequency Quasi-Periodic Oscillations (LFQPOs) have been associated with the position along the $\mathrm{Z}$ : the HBOs (horizontal branch oscillations), the NBOs (normal branch oscillations), and the FBOs (flaring branch oscillations).

NBOs with frequencies between 4.5 and $7 \mathrm{~Hz}$ have been reported from all $\mathrm{Z}$ sources. Their fractional rms amplitude is typically between 1 and $3 \%$ in the $1-10 \mathrm{keV}$ band, and are strongest near the middle of the NB. FBOs were seen only in two sources (Sco X-1, Priedhorsky et al. 1986; GX 17+2, Homan et al. 2002), while a broad excess that may be due to an FBO peak moving rapidly in frequency as the source moves rapidly up and down the FB has been reported in other sources (see e.g. GX 5-1, Jonker et al. 2002). FBOs occur on a small part $(\sim 10 \%$ of the total extent) of the Flaring Branch (FB) nearest the NB, and have frequencies in the range $\sim 6-25$ $\mathrm{Hz}$, increasing from the NB-FB vertex through the FB. With increasing frequency, the fractional amplitude of the FBOs remains approximately constant, while its width increases until the peak becomes too broad to distinguish from the broad band noise in the power density spectrum (the so-called high frequency noise, $\mathrm{HFN}$ ).

NBOs and FBOs are thought to be physically related, since in Sco X-1 the NBO frequency joins the FBO frequency as the source moves from the NB to the FB. However, the increase from $\sim 6 \mathrm{~Hz}$ to $\sim 10 \mathrm{~Hz}$ occurs in a very short segment of the $\mathrm{Z}$ track located just at the vertex of the NB-FB junction, and is at present still unresolved (Dieters \& van der Klis 2000). The transition is even less clear in GX 17+2; Homan et al. (2002) found that near the NB-FB vertex, the frequency of the FBO was a factor of $\sim 2$ higher than that of the NBO, which could mean that the NBO and FBO are harmonically related. However, by inspecting all single dynamical power spectra of observations with values of the curvilinear coordinate $S_{z}$ (tracking the position along the $\mathrm{Z}$ pattern, see van der Klis 1995) around 2 (corresponding to the NB-FB vertex), they found in some cases QPOs with intermediate frequencies $(\sim 10 \mathrm{~Hz})$, suggesting that the frequency does not jump directly from $\sim 7$ to $\sim 14 \mathrm{~Hz}$. However, no clear transitions were found.

At present, it is currently believed that NBOs and FBOs are different manifestations of the same phenomenon, the properties of which rapidly change at around the position of the lower vertex in the $\mathrm{Z}$ track. However, the transition between them has not yet been unambiguously resolved. In this paper we present the analysis of an RXTE observation of Sco X-1 in which, for the first time, a clear continuous fast transition from a Normal Branch Oscillation to a Flaring Branch Oscillation is seen and can be associated with the NB-FB vertex in the $\mathrm{Z}$ track. 
Table 1. RXTE/PCA data modes active during the Sco X-1 observation.

\begin{tabular}{lccc}
\hline \hline $\begin{array}{l}\text { Mode } \\
\text { Name }\end{array}$ & $\begin{array}{c}\text { Time res. } \\
(\mathrm{s})\end{array}$ & $\begin{array}{c}\text { Number of } \\
\text { PHA Channels }\end{array}$ & $\begin{array}{c}\text { PHA } \\
\text { Energy range (keV) }\end{array}$ \\
\hline Standard1 & $2^{-3}$ & 1 & $2-60$ \\
Standard2 & $2^{4}$ & 128 & $2-60$ \\
Binned1 & $2^{-13}$ & 1 & $2-32$ \\
Binned2 & $2^{-8}$ & 16 & $2-60$ \\
SB & $2^{-13}$ & 1 & $2-60$ \\
\hline
\end{tabular}

Table 2. Channel and energy boundaries of the soft and hard colors.

\begin{tabular}{ccc}
\hline \hline Color & Channels & Energy $(\mathrm{keV})$ \\
\hline Soft & $12-16 / 0-11$ & $4.4-6.2 / 2.0-4.4$ \\
Hard & $26-63 / 17-25$ & $9.4-23.3 / 6.2-9.4$ \\
\hline
\end{tabular}

\section{Data analysis}

We analyzed an RXTE/PCA observation of Sco X-1 from the RXTE public archive made on MJD 50230 (1996-05-27). The observation consists of three continuous data intervals of $\sim 1600, \sim 550$, and $\sim 2000$ s duration, respectively. All five PCA units were on during the whole observation. The PCA data were obtained in several simultaneous different modes (see Table 1). During the first interval, the pointing direction was offset by $\sim 0.3 \mathrm{deg}$, while in the other two intervals the offset was reduced to $\sim 0.005 \mathrm{deg}$. For this reason, we could not build the expected Z-track across the whole observation. We concentrated our analysis on the first of the three data intervals, during which a variable low frequency $(\sim 6-15 \mathrm{~Hz})$ QPO was detected.

We used Standard2 data to produce a color-color diagram (CD). For each $16 \mathrm{~s}$ data segment (the intrinsic resolution of the Standard2 mode), we defined two colors as ratios of count rates in two different energy bands. The energy bands used for the colors (soft and hard color) are given in Table 2. The CD is shown in Fig. 1: Sco X-1 was in its Flaring Branch (FB) at the beginning of the observation, and moved into the Normal Branch (NB) after $\sim 200 \mathrm{~s}$.

Since the $\mathrm{Z}$ track is not complete, we could not use the usual rank number $S_{z}$ used in the literature, which is defined using both vertices (Hasinger et al. 1990). In order to check the presence of a QPO along the Z track, we divided the CD in 14 intervals and calculated a power spectrum (with a Nyquist frequency of $128 \mathrm{~Hz}$ ) for each of them by averaging power spectra calculated every $16 \mathrm{~s}$ data interval. The two power spectra corresponding to the extremes of the $\mathrm{CD}$ (\#1 and \#14), together with three power spectra approximately corresponding to the vertex (\#9, \#10, and \#11) are shown in Fig. 2.

We then fitted each power spectrum with a combination of a simple power law and a broad Lorentzian shape, approximating the broad band noise, and a narrow Lorentzian shape, approximating the QPO peak. For the intervals in the FB, an additional narrow $(\Delta v / v \gtrsim 1)$ Lorentzian shape was needed, in order to better approximate the asymmetric profile of the FBO peak. PDS fitting was carried out with the standard Xspec

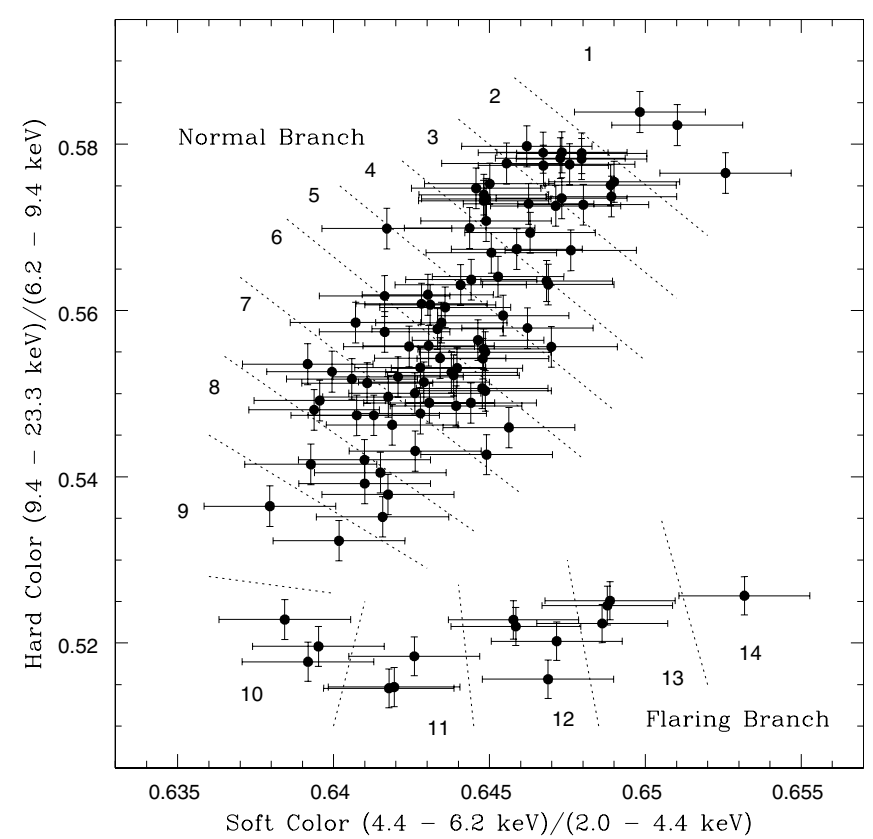

Fig. 1. Color-color diagram of the first $\sim 1600$ s. Time resolution is $16 \mathrm{~s}$. Dotted lines indicate the interval selection for the timing analysis.

fitting package, by using a one-to-one energy-frequency conversion and a unit response. Because of the low intensity of the broad band noise, the parameters of the power law and those of the broad Lorentzian were not well constrained, and their behavior could not be studied. However, since the intensity of the QPO peak was high in all intervals, the uncertainties in the underlying continuum did not affect the estimate of the peak parameters much.

In the upper panel of Fig. 3 we plot the QPO centroid frequency as a function of the interval number. The evolution of the QPO peak is evident, as the centroid frequency was $\sim 6 \mathrm{~Hz}$ when the source was in the upper part of the visible NB, then it slowly increased up to $\sim 7.5 \mathrm{~Hz}$ when the source approached the FB-NB vertex, and then quickly increased up to $\sim 15 \mathrm{~Hz}$ in the FB. In the bottom panel of Fig. 3, the total fractional rms and the QPO fractional rms are shown as a function of the interval number. Both slowly increase through the NB towards the vertex, and reach their maximum in the FB. The total rms slightly decreases after the vertex, while the QPO rms is consistent with remaining constant.

The transition appears to be very fast, as a result of the fast passage of the source through the NB-FB vertex. In order to track the QPO frequency as a function of time more finely, we produced a dynamical power spectrum for the whole observation by calculating a power spectrum every 4 seconds over PCA channels 0-35 (corresponding approximately to the $2-13 \mathrm{keV}$ energy range) and with a time resolution of $1 / 128 \mathrm{~s}$ (corresponding to a Nyquist frequency of $64 \mathrm{~Hz}$ ). The dynamical power spectrum and the correspondent light curve of the first $500 \mathrm{~s}$ of the observation, when the frequency transition occurred, are shown in Fig. 4.

The transition is clearly visible in the dynamical power spectrum. The QPO peak at the beginning of the observation (when the source is in its FB) is at frequencies $\sim 14 \mathrm{~Hz}$, has a 


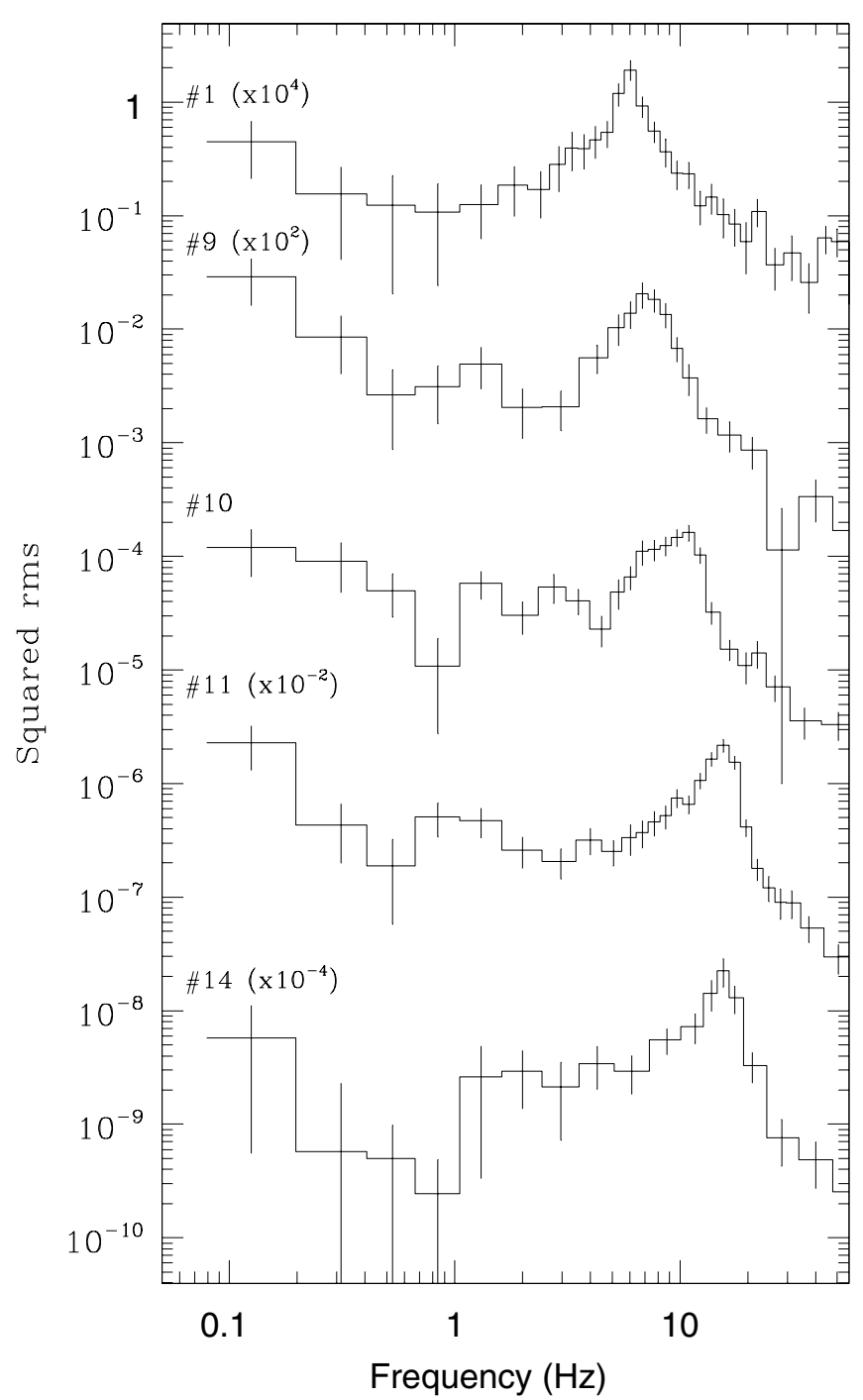

Fig. 2. Five power spectra corresponding to the extremes (\#1 and \#14) and to the vertex (\#9, \#10 and \#11) of the CD. Spectra are shifted by the indicated factors for clarity.

maximum at $\sim 15 \mathrm{~Hz}$ after less than 100 seconds, and rapidly decreases (corresponding to the FB-NB vertex) after a few hundred seconds to $\sim 6 \mathrm{~Hz}$. The peak then remains visible in the dynamical power spectrum at $\sim 6 \mathrm{~Hz}$ in the remaining part of the observation, during which the source is in the NB.

\section{Discussion}

We presented the first continuous monitoring of a rapid transition between Flaring Branch and Normal Branch Oscillations. Even though the transition could not be continuously tracked through the $\mathrm{Z}$ pattern in the $\mathrm{CD}$, the high statistics of the data makes it visible in the dynamical power spectrum, where it is resolved to take place in $\sim 100 \mathrm{~s}$.

The presence of the NBO/FBO in the $\mathrm{Z}$ sources has often been related to the fact that these sources accrete at nearEddington mass accretion rates. It is thought that at these high mass accretion rates a significant fraction of the accretion flow is in the form of a thick, perhaps nearly spherical flow. Also, the effects of radiation pressure are thought to play an important
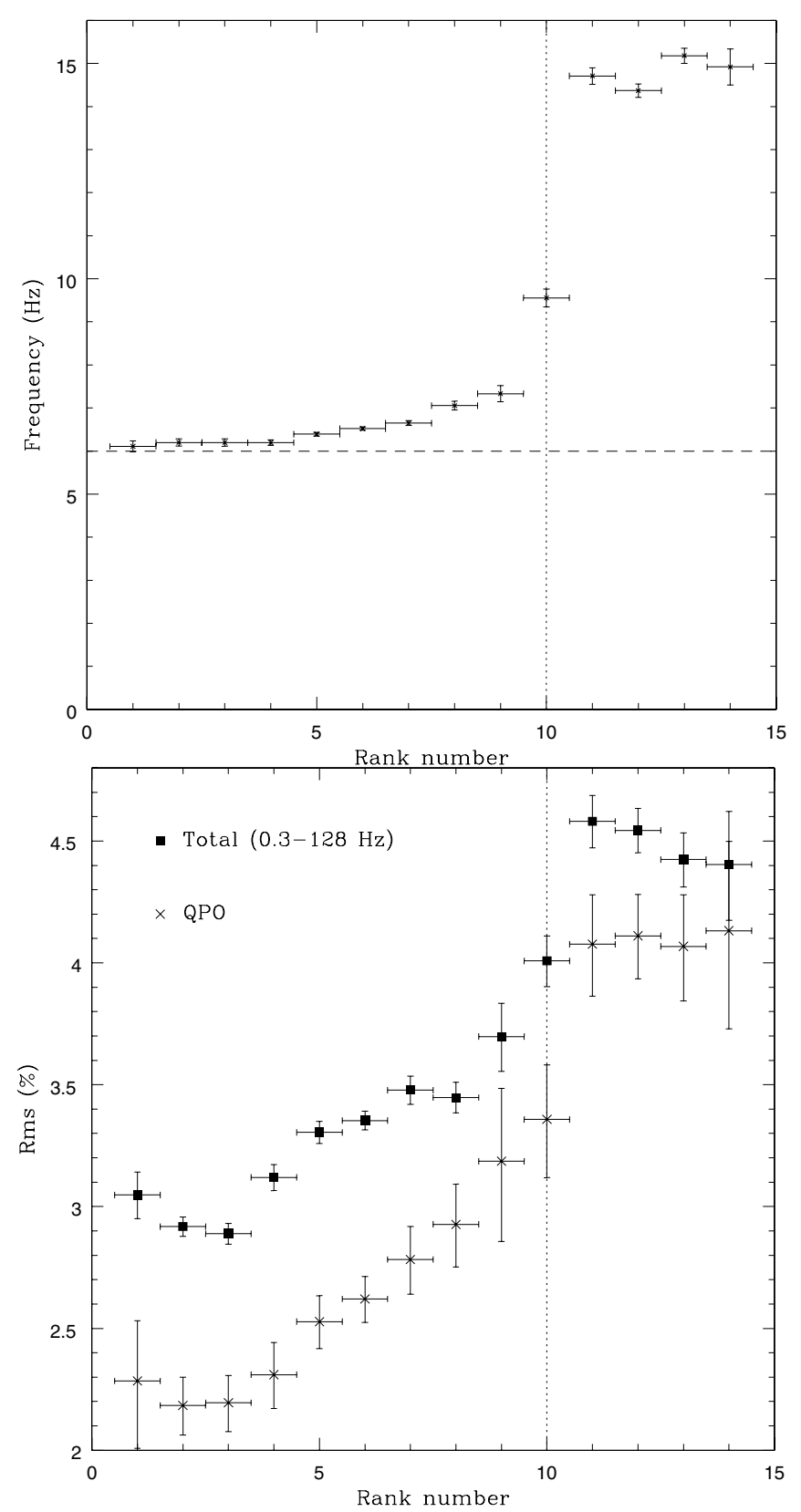

Fig. 3. QPO centroid frequency (upper panel) and fractional rms of the QPO and of the whole power spectrum (bottom panel) as a function of the interval number through the color-color diagram. The horizontal line in the upper panel indicates the " $6 \mathrm{~Hz}$ asymptote". The vertical line in both panels corresponds to the vertex in the $\mathrm{Z}$ track (see Fig. 1)

role in this regime. Fortner et al. (1989) suggest that the NBOs are the result of a radiation force/opacity feed-back mechanism within a spherical flow region. For reasonable physical parameters, the frequency of the NBOs is predicted to be $\sim 6 \mathrm{~Hz}$ at the onset of the process, and then to increase to $\sim 10 \mathrm{~Hz}$ as the luminosity approaches $L_{\text {Edd }}$. These oscillations can furthermore excite other modes with similar frequencies, which are expected to increase as the luminosity approaches and then exceeds $L_{\text {Edd }}$. However, it is not clear if the similarity of these two oscillation types can be as high as required to explain the smoothness of the transition reported in Sco X-1 (see Fig. 4). Another model for the NBOs was proposed by Alpar et al. (1992), in 


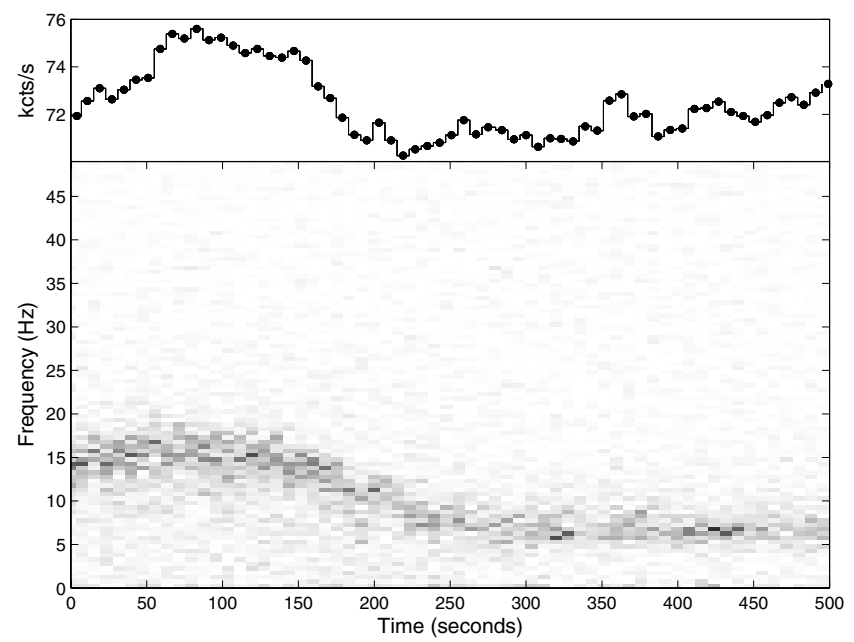

Fig. 4. 2-13 keV light curve (upper panel) and dynamical power spectrum (bottom panel) of the first $500 \mathrm{~s}$ of the observation.

which the NBO frequency is basically that of sound waves in a thickened accretion disk. However, this model does not explain how the NBO changes into the FBO. A strong challenge for these models is the recent discovery of $\sim 6 \mathrm{~Hz}$ QPOs with properties similar to those of NBOs in atoll sources (see e.g. Wijnands et al. 1999; Wijnands \& van der Klis 1999; Belloni et al. 2004), in which the mass accretion rate is always lower than the Eddington limit.

Casella et al. (2005) recently reviewed and further extended the similarities between the LFQPOs observed in $\mathrm{Z}$ sources and the three main types of LFQPOs observed in Black-Hole Candidates (BHCs, see the cited work and references therein), and proposed a one-to-one association where the C-, B-, and AType observed in BHCs correspond, respectively, to the HBO, $\mathrm{NBO}$, and FBO observed in $\mathrm{Z}$ sources. Whatever the physical mechanisms that determine these oscillations are, the presence of such mechanisms in both types of compact objects would clearly favor a disk origin for these oscillations, ruling out all models that involve any interaction with the surface or the magnetosphere of the neutron star (see e.g. Stella et al. 1999; see also Kluzniak 2004).

In this context, the smooth continuous transition between the NBOs and the FBOs appears to be very different from the fast, unresolved transitions observed between type-B and typeA QPOs in BHCs (Nespoli et al. 2003; Casella et al. 2004), i.e. the equivalent of NBOs and FBOs in this analogy. At present it is in fact not clear whether type-B and type-A QPOs originate from the same physical phenomenon, the properties of which can change in a short time scale (less than a few tens of seconds in the known cases), or if they are two distinct phenomena, one of which (type-A) is too weak to be detected when the other (type-B) is present (see e.g. Nespoli et al. 2003). Available data at present do not permit us to address this issue, and more observations (and/or better statistics) are needed.

Acknowledgements. This work was partially supported by the Italian Ministry of Education, University and Research under CO-FIN grants 2002027145 and 2003027534.

\section{References}

Alpar, M. A., \& Shaham, J. 1985, Nature, 316, 239

Alpar, M. A., Hasinger, G., Shaham, J., \& Yancopoulos, S. 1992, A\&A, 257, 627

Belloni, T., Parolin, I., \& Casella, P. 2004, A\&A, 423, 969

Casella, P., Belloni, T., Stella, L., \& Homan, J. 2004, A\&A, 426, 587

Casella, P., Belloni, T., \& Stella, L. 2005, ApJ, accepted [arXiv: astro-ph/0504318]

Dieters, S. W., \& van der Klis, M. 2000, MNRAS, 311, 201

Fortner, B., Lamb, F. K., \& Miller, G. S. 1989, Nature, 342, 775

Hasinger, G., \& van der Klis, M. 1989, A\&A, 225, 79

Homan, J., van der Klis, M., Jonker, P. G., et al. 2002, ApJ, 568, 878

Jonker, P. G., van der Klis, M., Homan, J., et al. 2002, MNRAS, 333, 665

Kluszniak, W. 2004, Rev. Mex. Astron. Astrofis., Serie de Conferencias, 20, IAU Coll., 194, 128

Nespoli, E., Belloni, T., Homan, J., et al. 2003, A\&A, 412, 235

Priedhorsky, W., Hasinger, G., Lewin, W. H. G., et al. 1986, ApJ, 306, L91

Stella, L., Vietri, M., \& Morsink, S. M. 1999, ApJ, 524, L63

van der Klis, M. 1995, in X-ray binaries, ed. Lewin, W. H. G., van Paradijs, J., \& van den Heuvel, E. P. J. (Cambridge: Cambridge University Press), 252

van der Klis, M. 2005, in Compact Stellar X-Ray Sources, ed. W. H. G. Lewin, \& M. van der Klis (Cambridge University Press), in press

Wijnands, R., \& van der Klis, M. 1999, ApJ, 522, 965

Wijnands, R., van der Klis, M., \& Rijkhorst, E. 1999, ApJ, 512, L39 